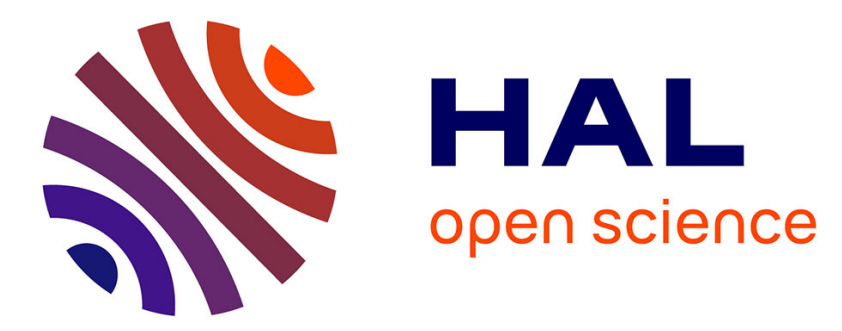

\title{
Deeply Virtual Compton Scattering on the neutron at JLab Hall A
}

\author{
M. Mazouz
}

\section{To cite this version:}

M. Mazouz. Deeply Virtual Compton Scattering on the neutron at JLab Hall A. 5th International Conference on Perspectives in Hadronic Physics, May 2006, Trieste, Italy. pp.41-48, 10.1016/j.nuclphysa.2006.10.048 . in2p3-00120353

HAL Id: in2p3-00120353

https://hal.in2p3.fr/in2p3-00120353

Submitted on 19 Dec 2006

HAL is a multi-disciplinary open access archive for the deposit and dissemination of scientific research documents, whether they are published or not. The documents may come from teaching and research institutions in France or abroad, or from public or private research centers.
L'archive ouverte pluridisciplinaire HAL, est destinée au dépôt et à la diffusion de documents scientifiques de niveau recherche, publiés ou non, émanant des établissements d'enseignement et de recherche français ou étrangers, des laboratoires publics ou privés. 


\title{
Deeply virtual Compton scattering on the neutron at JLab Hall A
}

\author{
M. Mazouz, for the Jefferson Lab Hall A Collaboration and the DVCS Collaboration ${ }^{\text {a }}$

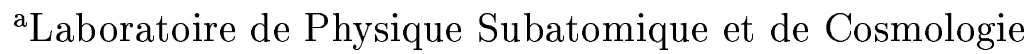 \\ IN2P3-CNRS/Université Joseph Fourier \\ 53 avenue des Martyrs 38026 Grenoble cedex, France
}

Deeply virtual Compton scattering (DVCS) is the simplest hard exclusive process to access generalized parton distributions (GPDs). In the case of a neutron this process is mostly sensitive to $E$, the least constrained GPD, which enters Ji's sum rule linking GPDs to the total quark angular momentum. We present the first dedicated DVCS experiment on the neutron which took place in the Hall A of Jefferson Lab in fall 2004. Experimental setup and preliminary results are discussed.

\section{INTRODUCTION}

During the past five decades, nucleon structure has been investigated through measurements of form factors and structure functions. From elastic electron scattering one discovered that the proton is not a point-like particle [1], and its charge and current distributions were inferred from the measurement of Dirac and Pauli electromagnetic form factors. From deep inelastic scattering one discovered quarks inside the nucleon [2], and after thirty years of research a rather complete mapping of quark momenta and spin distributions has been established. These two different but complementary approaches have similar limitations. The form factors do not exhibit any information about quark dynamics, while parton distributions do not provide any information about their spatial distribution.

In the late 90's, a new theoretical tool was developed linking these two representations of the nucleon. GPDs $[3,4]$ offer correlation information between different nucleon states in terms of partons, and allow via deep exclusive scattering for a more complete description of the nucleon than ever before. DVCS on the nucleon is the simplest hard exclusive process involving GPDs. Its amplitude gives access to $H, \tilde{H}, E$ and $\tilde{E}$, the four GPDs describing the non-perturbative structure of the nucleon. While the proton DVCS experiments are mostly sensitive to $H$ and $\tilde{H}$ because of the specific weighting of the observables, a neutron DVCS experiment is mostly sensitive to $E$, the least constrained GPD. It is essential to access the $E$ GPD not only because it is one of the ingredients in our understanding of the nucleon structure, but also because it enters on equal footings with $H$ in Ji's sum rule leading to the total angular momentum carried by quarks in the nucleon [3]. An exploratory DVCS experiment on the neutron [5] has taken place in Hall A of Jefferson Lab with the goal of measuring the beam-helicity-dependent DVCS cross-sections. The first section of this paper presents a brief overview of the DVCS theory and the kinemat- 
ics definition. The experimental setup and preliminary results are then discussed in the following sections before deducing some guidance for future DVCS experiments in Hall A.

\section{DEEPLY VIRTUAL COMPTON SCATTERING ON THE NEUTRON}

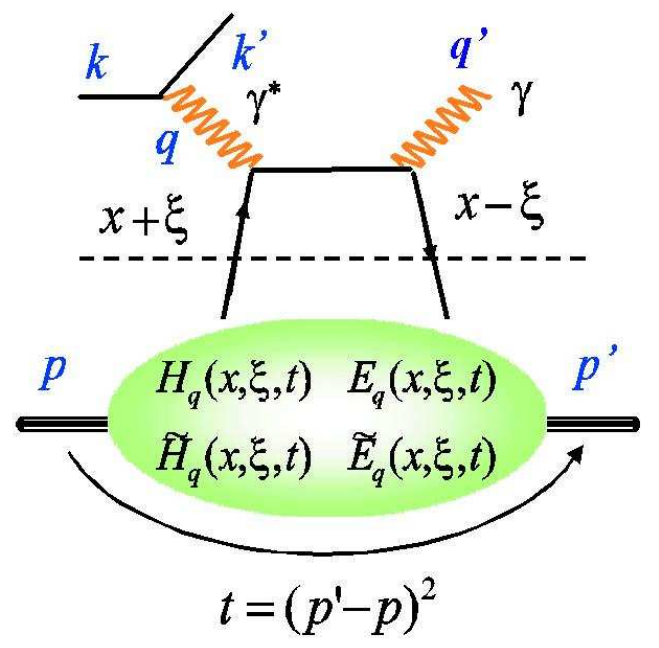

Figure 1. Handbag diagram for DVCS.

The lowest order QED diagram for virtual Compton scattering (VCS) corresponds to the absorption of a virtual photon of momentum $q$ by a nucleon of momentum $p$, producing an outgoing real photon of momentum $q^{\prime}$ and a nucleon recoiling with momentum $p^{\prime}$. DVCS is defined in the deeply virtual kinematics region, namely the Bjorken limit: $Q^{2}=$ $-q^{2} \rightarrow \infty$ and $Q^{2} / p . q$ finite. The leading diagram for this kinematics, the so-called handbag diagram (Figure 1), can be factorized [6] into a hard scattering kernel and a non-perturbative part described by GPD. This is depicted in Figure 1 where a quark with a momentum fraction $x+\xi$ absorbs a virtual photon, becomes highly off-shell, and then falls back to the nucleon ground state with a momentum fraction $x-\xi$ by radiating a real photon. To leading order in $\alpha_{s}$, the DVCS amplitude is given by

$\mathcal{T}^{D V C S} \propto \int_{-1}^{+1} \frac{G P D(x, \xi, t)}{x-\xi+i \epsilon} d x=\mathcal{P} \int_{-1}^{+1} \frac{G P D(x, \xi, t)}{x-\xi} d x-i \pi G P D(x=\xi, \xi, t)$

where $t=\left(p^{\prime}-p\right)^{2}$ is the momentum transfer to the nucleon. Note that GPDs enter the DVCS amplitude as an integral over $x$, then appearing in the real part through a principle value integral over $x$ and in the imaginary part through a fixed value at $x=\xi$. Experimentally, the DVCS process is accompanied by the Bethe-Heitler (BH) process where a real photon is emitted by the incoming or outgoing electron. Taking advantage of the interference between the Bethe-Heitler and DVCS amplitudes, the imaginary part of GPDs can be accessed from the difference between polarized beam cross sections

$\vec{\sigma}-\overleftarrow{\sigma}=2 . \mathcal{T}^{B H} \cdot \Im m\left(\mathcal{T}^{D V C S}\right)+\left[\left|\overrightarrow{\mathcal{T}}^{D V C S}\right|^{2}-\left|\overleftarrow{\mathcal{T}}^{D V C S}\right|^{2}\right]$ 
Moreover, the unpolarized cross section is related to the real part of the DVCS amplitude and therefore to an integral of GPDs over $x$.

$\sigma=\left|\mathcal{T}^{B H}\right|^{2}+2 . \mathcal{T}^{B H} . \Re e\left(\mathcal{T}^{D V C S}\right)+\left|\mathcal{T}^{D V C S}\right|^{2}$

The general expression of helicity-dependent cross sections in the differential phase space element $d^{5} \Phi=d Q^{2} d x_{B j} d \phi_{e} d t d \phi_{\gamma \gamma^{*}}$ where $\phi_{e}$ is the azimuthal angle of the scattered electron and $\phi_{\gamma \gamma^{*}}$ is the angle between the leptonic and hadronic planes, is given by [7]

$$
\begin{aligned}
\frac{d^{5} \Sigma}{d^{5} \Phi} & =\frac{1}{2}\left[\frac{d^{5} \vec{\sigma}}{d^{5} \Phi}-\frac{d^{5} \overleftarrow{\sigma}}{d^{5} \Phi}\right] \\
& =\sin \left(\phi_{\gamma \gamma^{*}}\right) \Gamma_{1}^{\Im} \Im m\left[C^{I}(F)\right]-\sin \left(2 \phi_{\gamma \gamma^{*}}\right) \Gamma_{2}^{\Im} \Im m\left[C^{I}\left(F^{e f f}\right)\right]+\frac{d^{5} \Sigma\left(|D V C S|^{2}\right)}{d^{5} \Phi}
\end{aligned}
$$

where, at the twist-3 approximation, the $\sin \left(\phi_{\gamma \gamma^{*}}\right)$ term is a twist-2 contribution (handbag diagram) and the $\sin \left(2 \phi_{\gamma \gamma^{*}}\right)$ is a twist-3 contribution. The $\Gamma_{n}^{\Im, \Re}$ are kinematic factors with a $\phi_{\gamma \gamma^{*}}$ dependence arising from the electron propagators of the $\mathrm{BH}$ amplitude. The $d^{5} \Sigma\left(|D V C S|^{2}\right) / d^{5} \Phi$ contribution is suppressed by at least one order of magnitude for the present kinematics. Finally, $\Im m\left[C^{I}(F)\right]$ is the observable to extract, with $\Im m\left[C^{I}\left(F^{e f f}\right)\right]$, which corresponds to a linear combination of three GPDs at $x=\xi$

$C^{I}(F)=F_{1}(t) \mathcal{H}(\xi, t)+\xi\left(F_{1}(t)+F_{2}(t)\right) \tilde{\mathcal{H}}(\xi, t)-\frac{t}{4 M^{2}} F_{2}(t) \mathcal{E}(\xi, t)$

$F_{1}$ and $F_{2}$ are the Dirac and Pauli form factors. The Compton form factors $\mathcal{H}, \tilde{\mathcal{H}}$ and $\mathcal{E}$ are a flavor combination of GPDs, for example:

$$
\begin{aligned}
\mathcal{H}(\xi, t) & =\sum_{f \in\{u, d, s\}}\left[\frac{e_{f}}{e}\right]^{2}\left\{i \pi\left[H_{f}(\xi, \xi, t)-H_{f}(-\xi, \xi, t)\right]\right. \\
& \left.+\mathcal{P} \int_{-1}^{+1} d x\left[\frac{1}{x-\xi}-\frac{1}{x+\xi}\right] H_{f}(x, \xi, t)\right\}
\end{aligned}
$$

In the proton case, the leading term of equation 5 is $F_{1}(t) \mathcal{H}(\xi, t)[8]$; in the neutron case, the leading term becomes $\frac{t}{4 M^{2}} F_{2}(t) \mathcal{E}(\xi, t)$ [8] since $\tilde{\mathcal{H}}$ is small by compensation of the $u$ and $d$ quark contributions and $F_{1}(t) \mathcal{H}(\xi, t)$ is also becoming small due to the Dirac form factor of the neutron. Therefore, DVCS on the neutron provides a simple way to access or constrain the $E$ GPD.

An exploratory experiment has taken place in Hall A which goal is the measurement of beam-helicity cross-section difference. This experiment followed a similar experiment on the proton which has shown strong indications of the handbag dominance at rather small $Q^{2}[9]$. Table 1 shows the kinematic setting. The neutron contribution is obtained from the difference between measurements on deuterium and hydrogen targets.

\begin{tabular}{lllllll}
\hline$s\left(\mathrm{GeV}^{2}\right)$ & $Q^{2}\left(\mathrm{GeV}^{2}\right)$ & $x_{B j}$ & $k^{\prime}(\mathrm{GeV} / c)$ & $\theta_{e}(\mathrm{deg})$ & $\theta_{\gamma *}(\mathrm{deg})$ & $\operatorname{Lum}\left(\mathrm{fb}^{-1}\right)$ \\
\hline 4.22 & 1.91 & 0.364 & 2.95 & 19.32 & 18.25 & 24000 \\
\hline
\end{tabular}

Table 1. Kinematics of the experiment; the luminosity is given for deuterium runs; $4365 \mathrm{fb}^{-1}$ is the corresponding value for hydrogen runs. 


\section{EXPERIMENTAL SETUP}

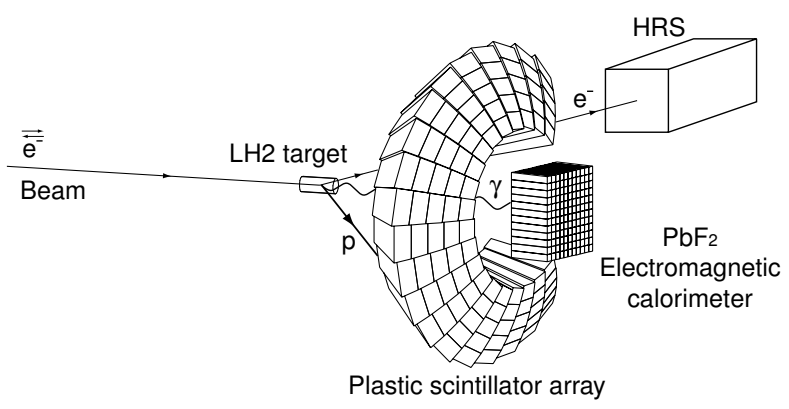

Figure 2. Schematic view of the DVCS detectors; the proton tagger detector, not shown here, is placed between the target and the proton array.

The data were acquired in Jefferson Lab Hall A. A $5.75 \mathrm{GeV}$ polarized electron beam was incident on a $15 \mathrm{~cm}$ liquid $\mathrm{H}_{2}$ or $\mathrm{D}_{2}$ target. The beam polarization was about $75 \%$ and the beam current of $\sim 4 \mu \mathrm{A}$ resulted in a luminosity of $4.10^{37} \mathrm{~cm}^{-2} \mathrm{~s}^{-1}$. Figure 2 shows the detectors used in this experiment. The scattered electron is detected in the Left High Resolution Spectrometer (L-HRS) [10] which has an angular acceptance of \pm 30 mrad horizontally, $\pm 60 \mathrm{mrad}$ vertically, and a momentum acceptance of $\pm 5 \%$. The precise measurement of the scattered electron accurately defines the virtual photon at fixed $Q^{2}$ and $x_{B j}$. The real photon is detected in an electromagnetic calorimeter $(11 \mathrm{x}$ 12 array of $3 \times 3 \times 18.6 \mathrm{~cm}^{3} \mathrm{PbF}_{2}$ crystals) centered around the virtual photon. With the measurement of the scattered electron and the real photon, the reaction kinematic is fully defined. To check the exclusivity of the reaction, the recoil nucleon is detected in an array of $5 \times 20$ scintillator blocks (Proton Array). A proton tagger detector, composed of two thin scintillator layers, is added in front of the Proton Array to distinguish neutrons from protons for scattering from the deuterium target. All the DVCS detectors, except the L-HRS, are placed at approximately $1 \mathrm{~m}$ from the target and centered at an angle of 18.25 degrees from the beam line. To minimize background, due to this direct view of the target at high luminosity, a $1 \mathrm{~cm}$ thick aluminum vacuum chamber was built surrounding the liquid target. In addition, specialized electronics are used to reduce the acquisition dead time and to deal with pile-up events in the detectors.

Each PMT from the electromagnetic calorimeter blocks is connected to a $1 \mathrm{GHz}$ Analog Ring Sampler (ARS) which acts like a digital oscilloscope [11]. It digitizes the signals with an analog memory chip in 128 values (one value per nanosecond). Figure 3 shows an example of ARS output representing two different pulses which can be separated offline with a wave form analysis. With electrons from elastic ${ }^{1} \mathrm{H}\left(e, e^{\prime} p\right)$ events detected in the $\mathrm{PbF}_{2}$ calorimeter at $k^{\prime}=4.2 \mathrm{GeV} / c$ we obtain a resolution of $2.4 \%$ in energy and 2 $\mathrm{mm}$ in transverse position. The ARS allow this resolution to be maintained even in the 
high background environment of the experiment. Figure 4 shows the timing coincidence spectrum between electrons and photons after ARS analysis.

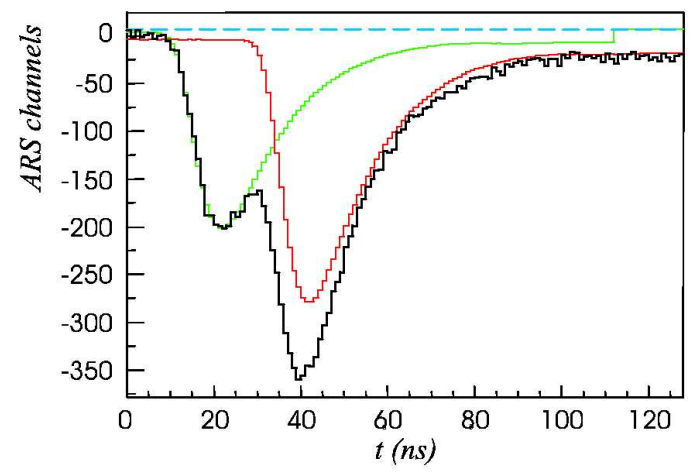

Figure 3. ARS output signal showing a two-pulse pile-up event.

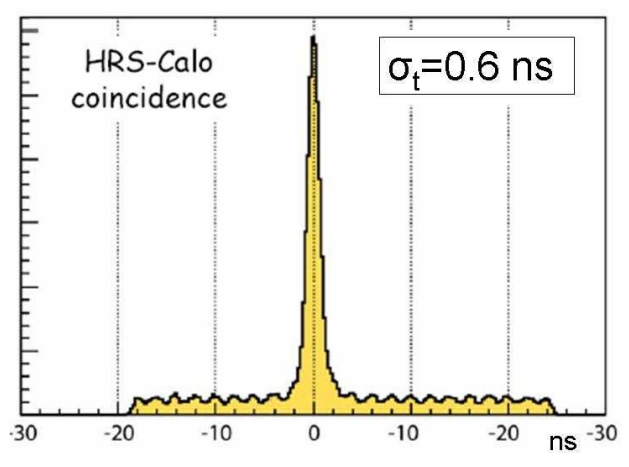

Figure 4. Timing coincidence spectrum between the L-HRS and the calorimeter. The 2 ns beam structure is visible with this high time-resolution.

A specific trigger is used to minimize the number of ARS channels for readout at each event, and hence the acquisition dead time. In fact, a trigger fast ADC digitizes all calorimeter signals within a $340 \mathrm{~ns}$ window. It starts by computing all overlapping sums of 4 adjacent blocks, then looks for at least one sum over a programmable threshold set to $1 \mathrm{GeV}$ equivalent. Finally, it validates or reject the event within 500 ns. A 3 GeV DVCS photon, for example, gives a 9 block cluster in the calorimeter with $90 \%$ of the energy deposited in a single block. Therefore, this trigger procedure allows to record only these 9 blocks (instead of 1 or 132).

\section{PRELIMINARY RESULTS}

The calibration of the calorimeter is monitored during the experiment by reconstructing the $\pi^{0} \rightarrow \gamma \gamma$ invariant mass from the analysis of ${ }^{1} \mathrm{H}\left(e, e^{\prime} \pi^{0}\right) X$ or ${ }^{2} \mathrm{H}\left(e, e^{\prime} \pi^{0}\right) X$ events. Figure 5 shows the invariant mass spectrum of two detected photons in the calorimeter and the missing mass squared of ${ }^{2} \mathrm{H}\left(e, e^{\prime} \pi^{0}\right) X$ events where the exclusive peak of the ${ }^{2} \mathrm{H}\left(e, e^{\prime} \pi^{0}\right) p$ reaction can be seen. Helicity-dependent and -independent cross-sections for exclusive $\pi^{0}$ electroproduction can also be extracted from these data [12].

The $\pi^{0}$ detection in the calorimeter is important, not only because it is a very reliable method to monitor the calibration, but also because it allows the evaluation and subtraction of the leading contamination source of DVCS. Figure 6 shows the missing mass squared of ${ }^{1} \mathrm{H}\left(e, e^{\prime} \gamma\right) X$ events and the different contamination sources. When two photons from a $\pi^{0}$ decay are oriented asymmetrically relative to the boost direction, a single photon carries most of its energy which is comparable to the DVCS photon energy. The detection of this photon in the calorimeter can lead to confusion with a DVCS event. By measuring the number of detected $\pi^{0}$ in symmetric decays, one can evaluate the total number of $\pi^{0}$ asymmetric decays and hence the contamination to subtract. 

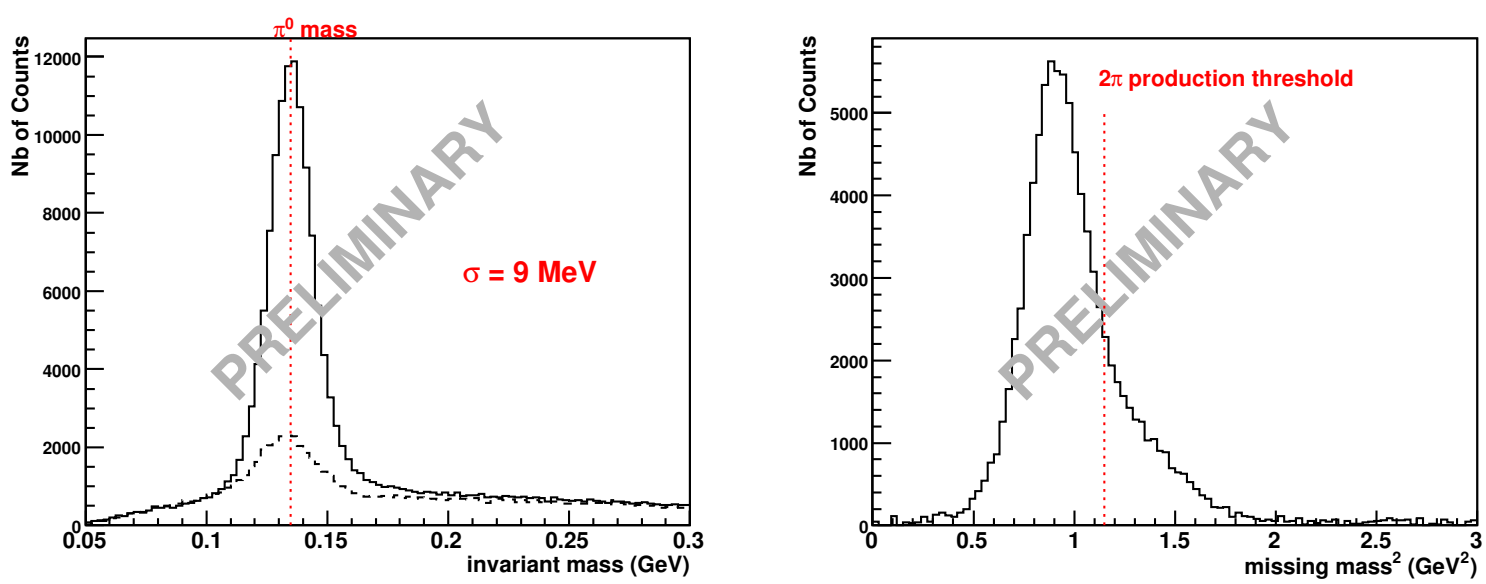

Figure 5. Left: invariant mass of two detected photons in the calorimeter; dashed spectrum represents accidentals. Right: missing mass square of $\left(\mathrm{e}^{\prime}, \pi^{0}\right)$ system; all detected $\pi^{0}$ are almost exclusive.

The final step is the selection of DVCS events in ${ }^{1} \mathrm{H}\left(e, e^{\prime} \gamma\right) X$ and ${ }^{2} \mathrm{H}\left(e, e^{\prime} \gamma\right) X$ reactions by putting a missing mass squared cut at the meson production threshold of $\left(M+m_{\pi}\right)^{2} \approx 1.15$ $\mathrm{GeV}^{2}$. With the recoil nucleon detectors, one can evaluate the contamination due to resolution effect by detecting the three DVCS particles in the final state. The resulting contamination of the exclusive yield by inclusive processes is below 3\%. Figure 7 shows the ${ }^{1} \mathrm{H}\left(e, e^{\prime} \gamma\right) X,{ }^{2} \mathrm{H}\left(e, e^{\prime} \gamma\right) X$ missing mass squared spectra and their difference yielding the neutron contribution. However, one should also consider the possible existence of a coherent deuteron contribution. In fact, there are theoretical indications [13] that the production of a real photon while keeping a deuteron in the final state can contribute significantly to the photon electroproduction cross section. To check this hypothesis, we plot the helicity-dependent number of counts $(\vec{N}-\overleftarrow{N})$ for two different regions of the missing mass (Figure 7, right). The results are shown in Figure 8. Due to our calculation of the missing mass squared of ${ }^{2} \mathrm{H}\left(e, e^{\prime} \gamma\right) X$ events, the coherent deuteron events are expected to be at $\sim M^{2}+t / 2$. Figure 8 shows that there are two contributions with two different asymmetry signs. The neutron asymmetry is of opposite sign to the proton asymmetry, as predicted by different models.

\section{CONCLUSION AND OUTLOOKS}

The DVCS experiment on the neutron is an exploratory experiment with the goal to test the possible existence of a DVCS signal on the neutron. In this experiment, we work at a precisely defined kinematics $\left(x_{B j}, Q^{2}\right.$ and $\left.s\right)$ and at a high luminosity, the detectors being in direct view of the target. Specialized electronics and equipment are used to minimize background and maintain a reasonable resolution during the experiment. The first conclusion is instrumental: with the L-HRS of Hall A and an electromagnetic calorimeter, one has enough resolution to select exclusive DVCS events and measure 


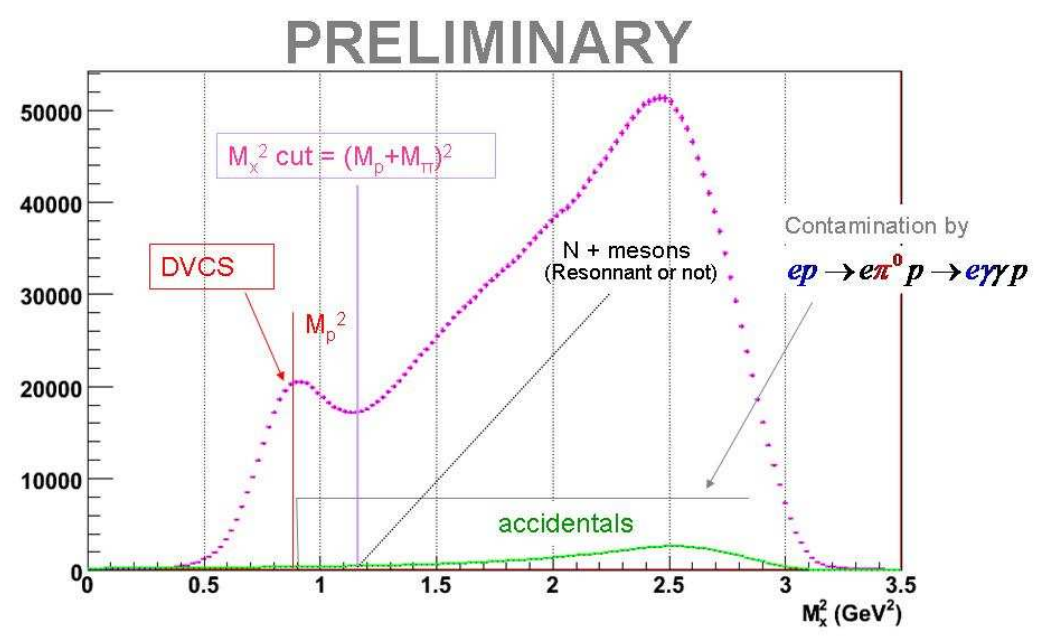

Figure 6. Missing mass squared of $\mathrm{H}\left(e, e^{\prime} \gamma\right) X$ events. The two sources of DVCS contamination are mesons production (starting at $\approx 1.15 \mathrm{GeV}^{2}$ ) and $\pi^{0}$ contamination (starting at the nucleon mass). Accidental contribution (green) is small.

the helicity dependence of DVCS cross sections. The second conclusion concerns the possibility to measure accurately a linear combination of GPDs and therefore constrain the $E$ GPD by studying DVCS on the neutron. The feasibility of an experimental approach comparing measurements from hydrogen and deuterium targets has been demonstrated. We also show that DVCS on the coherent deuteron can be measured at the same time by this method.

This experiment is to be followed by other future experiments utilizing the same simple equipment. A larger kinematics mapping is needed to get more information and hence more constraints on the neutron and the nucleon GPD in general. This effort will lead to a more complete comprehension of the nucleon properties such as the composition of nucleon spin.

\section{REFERENCES}

1. R. Hofstadter, Ann. Rev. Nucl. Sci. 7 (1958) 231.

2. J. Friedman and H. Kendall, Ann. Rev. Nucl. Sci. 22 (1972) 203.

3. X.D. Ji, Phys. Rev. Lett. 78 (1997) 610.

4. D. Mueller, D. Robaschik, B. Geyer, F. M. Dittes, and J. Horejsi, Fortschr. Phys. 42 (1994) 101; A.V. Radyushkin, Phys. Rev. D56 (1997) 5524.

5. P. Y. Bertin, C. Hyde-Wright, F. Sabatié, E. Voutier et al., Jefferson Lab Proposal E03-106 (2003).

6. M. Diehl, T. Gousset, B. Pire and J. P. Ralston, Phys. Lett. B411 (1997) 193; X.D. Ji and J. Osborne, Phys. Rev. D58 (1998) 094018.

7. A.V. Belitsky, D. Mueller, and A. Kirchner, Nucl. Phys. B629 (2002) 323.

8. Model following K. Goeke, M.V. Polyakov and M. Vanderhaeghen, Prog. Part. Nucl. Phys. 47 (2001) 401. Implementation by L. Mossé and adaptation to the neutron by 

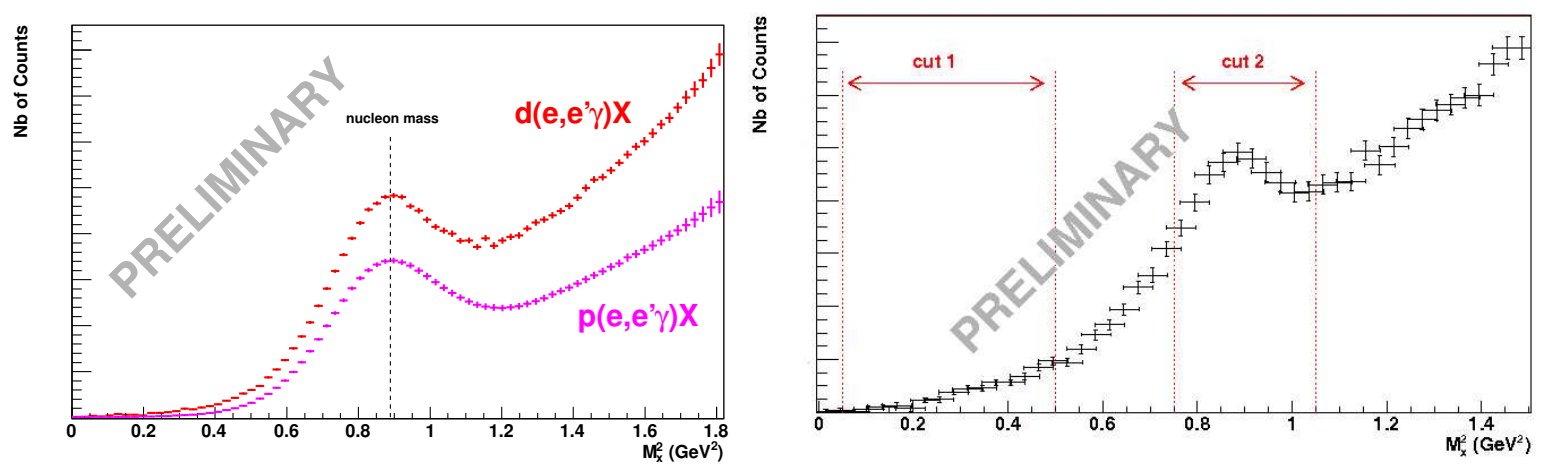

Figure 7. Left: missing mass squared of $\mathrm{H}\left(e, e^{\prime} \gamma\right) X$ and ${ }^{2} \mathrm{H}\left(e, e^{\prime} \gamma\right) X$. Right: difference between the two previous spectra giving neutron and the coherent deuteron contributions; one expect a dominance of coherent deuteron events in the cut-1 region and a dominance of neutron events in the cut-2 region.
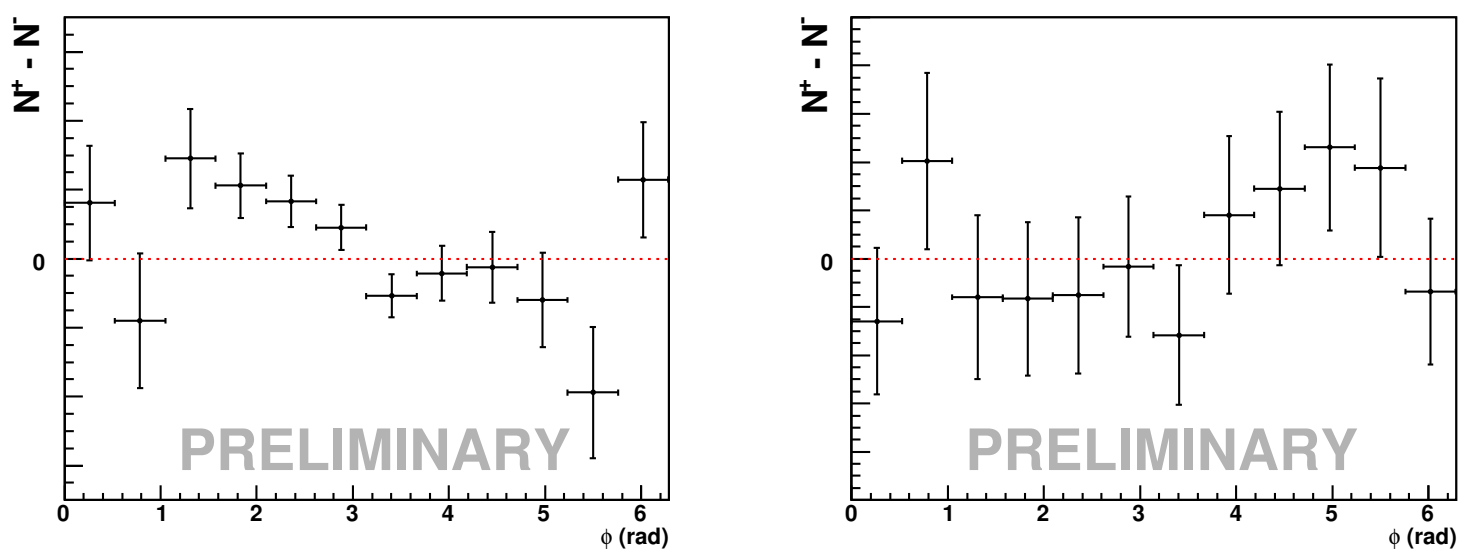

Figure 8. Left: counting asymmetry (arbitrary units) in the region where we expect a dominance of coherent deuteron events. Right: counting asymmetry in the region where we expect a dominance of neutron events.

F. Sabatié.

9. C. Muñoz-Camacho et al., to be published.

10. A. Alcorn et al., Nucl. Inst. and Meth. A 522, 294 (2004).

11. F. Feinstein, Nucl. Inst. and Meth. A 504, 258 (2003).

12. A. Camsonne, Doctorat Thesis, Université Blaise Pascal, France (2005).

13. F. Cano, B. Pire, Eur. Phys. J. A 19 (2004) 423; A. Kirchner, D. Müller, Eur. Phys. J. C 32 (2004) 347. 